\title{
Correction
}

\section{MEDICAL SCIENCES}

Correction for "Hypoxia treatment reverses neurodegenerative disease in a mouse model of Leigh syndrome," by Michele Ferrari, Isha H. Jain, Olga Goldberger, Emanuele Rezoagli, Robrecht Thoonen, Kai-Hung Chen, David E. Sosnovik, Marielle Scherrer-Crosbie, Vamsi K. Mootha, and Warren M. Zapol, which appeared in issue 21, May 23, 2017, of Proc Natl Acad Sci
USA (114:E4241-E4250; first published May 8, 2017; 10.1073/ pnas.1621511114).

The authors note that Fig. 2 and its legend appeared incorrectly. Additionally, the legends for Figs. 8 and 9 appeared incorrectly. The corrected figures and their corrected legends appear below. The online version has been corrected.
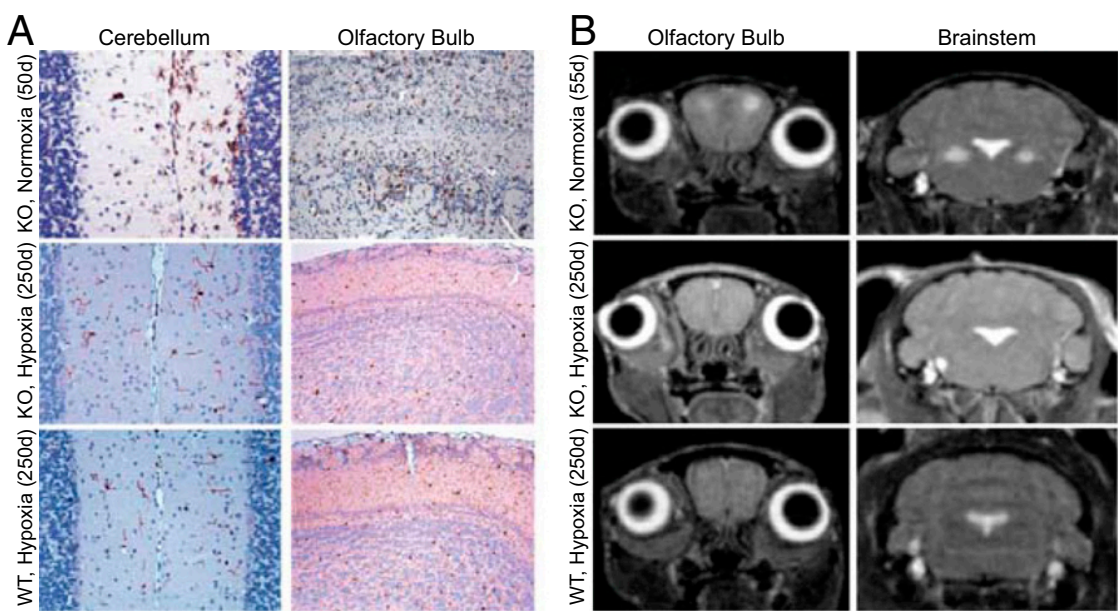

Fig. 2. Absence of neurodegenerative pathology in 250-d-old hypoxia-treated Ndufs4 KO mice. (A) Representative images with staining for the microglial activation marker Iba-1 $(n=3)$. Normoxic KO mice at $50 \mathrm{~d}$ show a significant inflammatory response in the cerebellum and OB. Analogous images in $250-\mathrm{d}$-old hypoxic KO mice and WT mice do not show brain inflammation. (B) Axial head MRI images showing bilateral, symmetrical hyperintense lesions in the OB (Left) and brainstem (Right) of normoxic-breathing KO mice at $55 \mathrm{~d}$. These lesions were not present in hypoxic KO mice at $250 \mathrm{~d}$. 


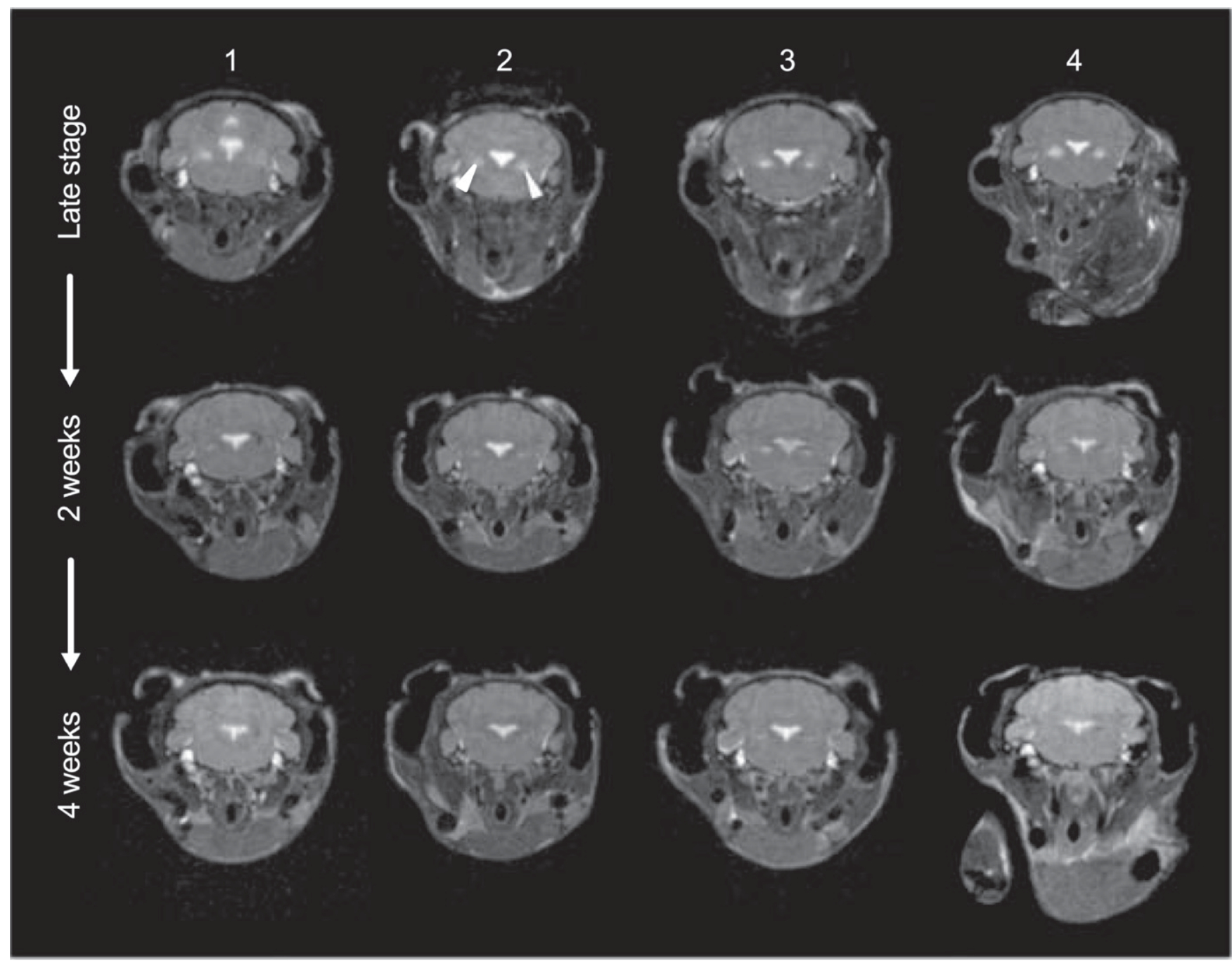

Fig. 8. Breathing $11 \% \mathrm{O}_{2}$ in late-stage neurological disease reverses the radiographic lesions of Ndufs4 mice seen on MRI. Four Ndufs4 mice were breathing $21 \% \mathrm{O}_{2}$ until they developed late-stage neurological disease $(55 \mathrm{~d})$. They underwent MRI to document bilateral lesions in the vestibular nuclei (Upper, white arrow). Subsequently they commenced breathing $11 \%$ oxygen. The mice were scanned again at $2 \mathrm{wk}$ and 4 wk of hypoxic breathing (Middle and Lower, respectively). Neurologic lesions disappeared by $4 \mathrm{wk}$ of hypoxic breathing. The section of the fourth ventricle shown at the center of the brainstem appeared enlarged in the early scans, suggesting parenchymal atrophy. After 4 wk of treatment, this area appeared to be reduced in size, and morphological relationships were restored. 


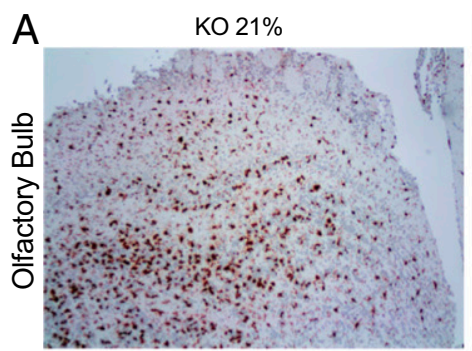

B $\quad$ KO $11 \%$

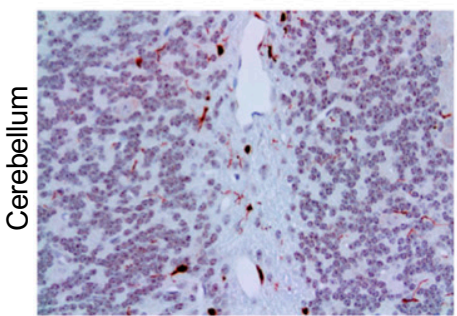

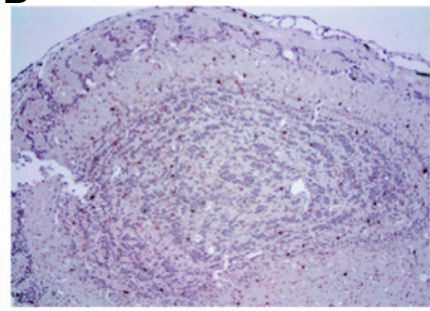

C

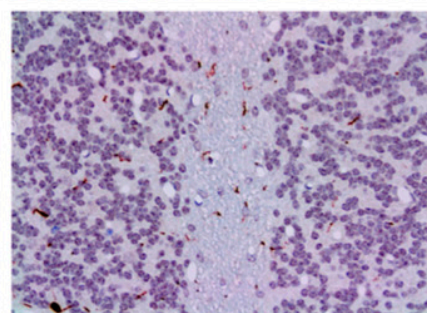

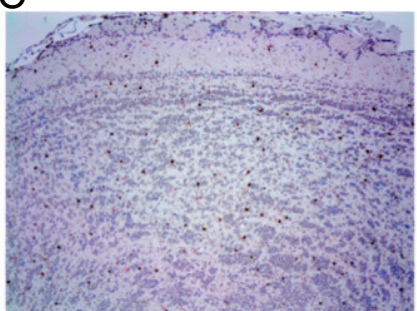

WT $21 \%$

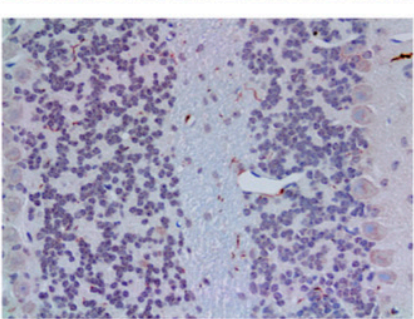

D WT $11 \%$
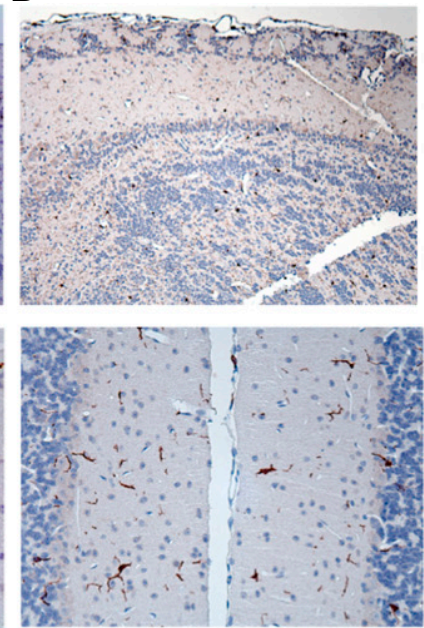

Fig. 9. Breathing $11 \% \mathrm{O}_{2}$ in late-stage neurological disease reverses pathological inflammation in the brains of Ndufs4 KO mice. Representative images with Iba-1 staining of the OB and cerebellum (CB) in KO mice and WT controls ( $n=3$ per group). Iba-1 is a marker of inflammation in the brain, indicative of microglial activation. Images demonstrate the reversibility of the neuropathological pattern by breathing $11 \% \mathrm{O}_{2}$ at the late stage of disease $(55 \mathrm{~d})$. ( $A$ ) $\mathrm{KO}$ mice breathing $21 \% \mathrm{O}_{2}$. $(B) \mathrm{KO}$ mice breathing $21 \% \mathrm{O}_{2}$ up to $55 \mathrm{~d}$ and then breathing $11 \% \mathrm{O}_{2}$ (to $160 \mathrm{~d}$ ). MRI-demonstrated reversal of the lesions reported in Fig. 8 was observed in these same mice. (C) Normoxic WT controls. (D) Hypoxic WT controls.

www.pnas.org/cgi/doi/10.1073/pnas.1708137114 